\title{
Kommunale Kommunikation in digitalen Zeiten
}

\author{
Andreas Möser
}

\section{Intro}

Nirgendwo agiert Politik näher am Volk als in Städten und Gemeinden. Jede Entscheidung eines kommunalen Parlamentes oder einer Verwaltung, jeder Behördenfehler, jedes Versagen einzelner Sachbearbeiter und jede technische Panne haben unmittelbar Folgen für den Alltag der Bürger. Entsprechend nah am Leben gestaltet sich die Kommunikation einer Kommunalverwaltung - oft anders als nach Lehrbuch konzipiert. Werfen wir deshalb exemplarisch einen Blick in die Praxis der kommunalen Kommunikation wie die der Landeshauptstadt Hannover, ergänzt um Beispiele aus anderen Städten unterschiedlicher Größe. Ob 50.000 oder 500.000 Einwohner, ob in der norddeutschen Tiefebene oder in höheren Lagen: Die Herausforderungen gleichen sich - ob in Hannover, Heidenheim oder Heidelberg.

Eines vorweg: Es geht stets sehr konkret um das Alltagsleben unseres Gemeinwesens. Die Themen reichen von A wie Abwasser- und Abfallbeseitigung über die öffentlichen Bäder, Kindergärten, Parkgebühren, Schulen, Straßensanierungen, Unterbringung und Integration von Flüchtlingen bis zum Wohnungsbau oder, soweit vorhanden, dem Wohl und Wehe eines städtischen Zoos. Erfolg und Misserfolg, Lob und Kritik sind davon abhängig, wie zuverlässig und effizient eine Kommunalverwaltung in all diesen Bereichen arbeitet. Schafft sie das nicht, hat das unmittelbare Folgen für die Kommunikationsteams. Diese sind je nach Einwohnerzahl, Finanzkraft und dem jeweiligen Willen der Verwaltung und ihres Bürgermeisters respektive

\footnotetext{
A. Möser $(\bowtie)$

Moeser Communications, Hannover/Ilsede, Deutschland

E-Mail: info@moeser-communications.de
} 
Oberbürgermeisters zur Öffentlichkeitsarbeit unterschiedlich ausgestattet. Für jede Kommune gilt: Der Wechsel von Wohlfühl-Kommunikation beim Fassanstich des alljährlichen Volks-, Oktober- oder Schützenfests, der Eröffnung einer neuen Fahrradstraße oder dem Spatenstich in einem Neubaugebiet hinüber in den Krisenmodus geschieht meist ruckartig, unverhofft und dennoch regelmäßig.

Wenn Eltern für ihre geliebten Kleinen keinen Krippenplatz in der Nähe finden, wenn ramponierte Schultoiletten wegen leerer Kassen und nachlässiger Kontrolle oder beidem seit Jahren nicht renoviert werden, wenn es an Parkplätzen in Wohnstraßen fehlt oder die Fußgängerampel vor dem Seniorenheim seit drei Tagen defekt ist, ließe sich das eigentlich in Ruhe klären und erklären. Doch da solcher Art Alltagsärger mittlerweile zuerst an das Leser-Telefon oder das E-Mail-Postfach der Lokalmedien gemeldet wird oder per Facebook oder Twitter gleich direkt öffentlich gemacht wird, anstelle zunächst die zuständige Verwaltung $\mathrm{zu}$ informieren, gerät das Kommunikationsteam ruckzuck in Erklärungsnot. Konfrontiert mit prompten Anfragen der Lokalmedien als Anwälte ihrer Leserschaft und reflexartigem Wegtauchen der Fachleute in den mutmaßlich zuständigen Fachbereichen der eigenen Kommunalverwaltung, ringen Pressesprecher mit dem Anspruch, schnell, ehrlich und umfassend zu kommunizieren, und der Realität, dass sich zunächst nichts Belastbares zusammentragen lässt außer den bereits mit Empörung öffentlich ausgebreiteten Problemtatsachen. Das Kommunikationsdrama nimmt seinen Lauf in einschlägigen Medienberichten, garniert mit dem gern verwendeten blassen Erstkommentar der Kommunalverwaltung, dass der reklamierte Sachverhalt wohl so stimme, die Ursachen und die Problembeseitigung noch genauer und natürlich schnellstmöglich und umfassend geprüft werde - magere Floskelkommunikation also, die niemanden zufrieden stellt, am wenigsten das Kommunikationsteam selbst. Derartige Alltags-Krisenkommunikation gehört zum täglichen Brot kommunaler Kommunikationsteams.

Dass dies zwangsläufig so ablaufen muss, sehen einschlägig erfahrene Kommunikationsberater in der Regel kritisch. Die hier als notorisch krisenhaft beschriebene Praxis kommunaler Kommunikation ist zweifellos vielschichtiger und damit differenzierter zu betrachten. Sie lässt sich mit klassischen Handbuch-Kommunikationskonzepten nur annähernd erfassen und bearbeiten. Denn die Rahmenbedingungen und Herausforderungen für die Kommunikation von Kommunalverwaltungen unterliegen einem radikalen Wandel, der seit dem Einzug des Internets und digitaler Techniken in alle Winkel unseres Alltags auch die Medien- und Kommunikationswelt kräftig durchschüttelt, sodass kein Stein auf dem anderen bleibt. So werden folgende Fragen aufgeworfen:

Wie wirkt sich die Digitalisierung der Kommunikation konkret auf die Kommunikation in Städten und Gemeinden aus? Was sind die Anforderungen an eine 
praxistaugliche aktive und krisensichere kommunale Kommunikationsstrategie? Was taugen neue Konzepte, wenn Bürger grundsätzlich kritischer, fordernder und bisweilen nur noch verdrossen auf ihre Kommunalverwaltungen blicken? Wie muss eine Kommunalverwaltung ihre Kommunikation neu begreifen, angesichts eines ungebremsten Reichweitenschwundes der Lokalmedien? Wie agiert kommunale Kommunikation angesichts der neuen Macht der Adressaten von Kommunikation, die per Shitstorm jede Verwaltung öffentlich vor sich hertreiben können, wie inszeniert oder zufällig auch immer? Im Rahmen dieser Fallstudie werden keine Allheilrezepte ausgestellt. Aus der Diagnose aktueller Probleme kommunaler Kommunikation kristallisieren sich allerdings einige konkrete Therapievorschläge heraus.

\section{Was tun? - Die digitale Revolution}

\subsection{Machtverschiebung per Internet}

Was den Alltag der öffentlichen Kommunikation radikal verändert hat, ist die fundamentale Machtverschiebung in öffentlichen Diskursen durch das Internet. Kommunikation auf der Einbahnstraße ist passé. Kommunikation fährt vielspurig auf der Datenautobahn mit reichlich Gegenverkehr. Der Bremer Psychologe, Unternehmer und Internetforscher Prof. Peter Kruse umriss 2010 vor der Enquete-Kommission des Deutschen Bundestages „Internet und digitale Gesellschaft“ dies eindrucksvoll mit dem Superlativ „Revolution“ (Kruse 2010, S. 6). Kruse veranschaulichte die grundlegende Machtverschiebung in der Kommunikation von den Anbietern der Information zu den Nachfragern.

Die rasant gewachsene Vernetzungsdichte mit der Möglichkeit für alle, spontan zu jeder Tages- und Nachtzeit auf öffentlichen Plattformen wie Facebook und Twitter zu kommunizieren und per Posts und Retweets Erregung zu produzieren, gibt allen im Netz aktiven Menschen Macht. Wenn Vernetzungsdichte, hohe Spontanaktivität und kreisende Erregung zusammenkommen, dann - so Kruse (2010) - haben diese Kommunikationssysteme eine Tendenz, sich bei einem Thema mit Erregungspotenzial gegenseitig hochzuschaukeln: „Dann werden diese Systeme plötzlich mächtig und zwar ohne, dass man vorhersagen kann, wann das genau passieren wird." (Kruse 2010, S. 6). Längst geht es im Internet nicht mehr um den Zugang zu Informationen oder darum, sich darzustellen und Spuren zu hinterlassen (Scheller und Ullrich 2018). Mittlerweile schließen sich Internet-Nutzer teils gezielt, teils spontan im Netz kommunikativ zusammen, lassen 
sich von Bloggern inspirieren und entwickeln auf diese Weise eine Macht, die sich nicht vorhersagen lässt (Neuberger 2018, S. 38 ff.).

Konnten und können Lokalzeitungen nur wenige Leserbriefe - und diese meist gekürzt - veröffentlichen, so bietet das Netz unbegrenzt Raum für Kommentare. Aus Kritik werden Erregungswellen, die sich im Extremfall zu öffentlichen Shitstorms aufschaukeln und die öffentliche Meinung dominieren.

\subsection{Die Medien - Schneller, flacher, tiefer in die Krise}

Führendes Medium in der Landeshauptstadt Hannover ist die zum Madsack-Verlag gehörende ,Hannoversche Allgemeine Zeitung' (HAZ). Doch die Auflagenkurve des Marktführers, wie die der beiden anderen Tageszeitungen in Hannover (,Neue Presse'/Madsack und BILD-Zeitung/Hannover-Ausgabe), kennt seit Jahren nur eine Richtung: abwärts. Auch bundesweit sinkt die verkaufte Auflage deutscher Tageszeitungen stetig, laut IVW-Angaben zuletzt um 4,5\%, von knapp täglich 16,4 Mio. verkaufter Blätter (Ende 3. Quartal 2017) auf gut 15,6 Mio. (Ende 3. Quartal 2018), ein Minus von 750.000 Zeitungen. In einem Jahr verschwindet die Auflage von sieben Tageszeitungen der Stärke der HAZ. Weder in Hannover noch anderswo können die Online-Angebote dies auch nur halbwegs kompensieren. Die gesamte Branche krankt zudem an dem Kardinalfehler, mit Einführung des Internets die journalistische Ware plötzlich verschenkt zu haben, um nur über Klickzahlen Reichweite zu erzielen und auf dieser Basis dann kostenpflichtig Online-Werbung einzutreiben. An dieses Geschäftsmodell glaubt heute niemand mehr in der Medienbranche (Kaube 2018, S. 93). Doch die nachhaltig selbst erzeugte Gratis-Mentalität einer ganzen Generation junger Internet-User lässt sich nicht so ohne weiteres wieder verändern.

Der wirtschaftliche Druck erzwingt eine nie gekannte Konzentration der Lokalzeitungslandschaft in künftig eine Handvoll großer Verlage. Madsack beherbergt mittlerweile 15 Tageszeitungen und erreicht nach eigenen Angaben über das eigens gegründete Redaktionsnetzwerk Deutschland (RND) täglich mehr als vier Millionen Leser. Der Konzentrationsprozess auf wenige Verlagsnetzwerke (neben Madsack/RND, u. a. die Funke-Gruppe, die Ippen-Gruppe und natürlich der Springer-Verlag) sichert vorläufig die Existenz vieler Lokalblätter, entlastet sie dennoch nicht von Personalabbau und Produktionsdruck in allen Kanälen. Das Ziel, die Abkehr der Kunden, zumindest zu bremsen, führt zu einer Veränderung der eigenen Rolle. Berichtet wird nicht mehr zwingend über das, was geschieht, sondern zunehmend über das, was der Leser möchte. Lokaljournalismus entwickelt sich vom kritischen Beobachter im Gemeinwohlinteresse 
zur politischen Willensbildung zum Interessensvertreter der eigenen Leserschaft. Lokalredaktionen fordern ihre Leser auf, Alltagsärger und Missstände der Redaktion zu melden - und damit unausgesprochen nicht zunächst der zuständigen Behörde wie etwa der Stadtverwaltung. Die Zeitung sieht sich als Anwalt der Bürger. Zwar besteht der Anspruch, Bürger-Beschwerden kritisch zu prüfen. Die Bürgersicht passt jedoch naturgemäß besser zur kritischen Grundhaltung von Medien. Wer Anwalt der Leser ist, recherchiert bei der Stadtverwaltung eher in dessen Interesse. Dies führt bisweilen zu der absurden Situation, dass eine Stadtverwaltung über die Medien mit Bürgern kommuniziert, anstatt unmittelbar.

Der Rollenwechsel vom kritischen Beobachter zum aktiven Mitgestalter der Geschickte einer Stadt manifestiert sich in regelmäßigen Foren oder mitunter auch Veranstaltungsreihen zu Zukunftsfragen der Stadtentwicklung, bei der HAZ etwa unter dem Motto „Übermorgen“, kurz nach einem intensiven offiziellen Stadtdialog des Oberbürgermeisters unter dem Titel „Mein Hannover 2030“ mit mehr als 15.000 Teilnehmern, der die Lokalmedien in der Intensität und der Vielzahl von Veranstaltungen allerdings überfordert hat. Ein selbst inszenierter „Stadtdialog“ ist ressourcenmäßig einfacher abzudecken, zudem exklusiv und ist damit vor allem ein vom Journalismus gestütztes Marketinginstrument. Zu Recht ist zu fragen, warum diese Bürgerbeteiligung durch einzelne Medien Erfolg hat, obgleich dies eine ureigene Aufgabe der Kommunalverwaltung ist, offen für alle und offen auch vor allem auch für alle Medien. Wie eine Stadtverwaltung die zweifellos schon seit einigen Jahrzehnten praktizierte Bürgerbeteiligung weiterentwickeln muss, wird im Verlauf der Fallstudie noch zu betrachten sein. Ein Grund für den Erfolg solcher Medienveranstaltungen liefern die Behörden allerdings selbst: Erst wenn Bürger Lokalmedien einschalten, kommt häufig in der Verwaltung etwas ins Rollen - dann meist hektisch, schlecht koordiniert und mit entsprechend holpriger Kommunikation.

\subsection{Die Bürger - „Die Lunte wird kürzer“}

Dass Beamte in Verwaltungen vermeintlich faul sind, spät kommen und früh gehen und dergleichen mehr, sind altbekannte Stammtisch-Urteile. Gelegentlich werden sie von der Praxis sogar bestätigt. Die Ausfallquote in Behörden ist indessen nicht höher als in Unternehmen. Kundenbefragungen in den Behörden haben zum Beispiel in Hannover in vielen Bereichen regelmäßig mehrheitlich positives Feedback ergeben. Mit der diffusen kritischen Grundstimmung ließe sich leben, wenn Probleme in Bürgersprechstunden oder per Beschwerde-App im Internet sachlich übermittelt würden. Ob am Behördenschalter, am Telefon oder 
per E-Mail haben es Verwaltungsbeschäftigte jedoch inzwischen oft mit einem rauen Ton, mit persönlichen Angriffen, Pöbeleien und Provokationen zu tun. „Die Lunte bei den Bürgern wird kürzer", formulierte es eine Stadtsprecherin auf der Herbstkonferenz kommunaler Kommunikationsexperten im Deutschen Städtetag im September 2018 in Heidenheim. Bürger greifen schneller zur Beschwerde, vergreifen sich häufiger im Ton, schreiben regelrechte „Hass-Mails“ - und gehen oft sofort an die Medien. So steigt die Zahl genervter, irritierter, frustrierter und zunehmend verängstigter Verwaltungskollegen. Eine in die Defensive gedrängte Verwaltung tut sich umso schwerer, aktiv zu kommunizieren.

Verschärft wird der respektlose Umgang mittels der sozialen Medien, wo ein Anwurf in Echtzeit ungefiltert auch von anonymen Usern veröffentlicht werden kann. Ein Verstoß gegen die Netiquette kann allenfalls im Nachhinein gerügt oder korrigiert werden, wenn überhaupt. Die Verwaltung gerät unmittelbar unter Druck, reagieren zu müssen und mit jeder Verzögerung mehr ins Hintertreffen.

Themen von Beschwerden sind alle denkbaren Alltagsdinge: Schlaglöcher, Müll in öffentlichen Grünanlagen, aggressives Betteln in der Fußgängerzone, offensichtliches Dealen mit Rauschgift in Parks, Lärm durch öffentliche Veranstaltungen, eine neue Blitzanlage im Straßenverkehr, Trinkgelage von Obdachlosen am Hauptbahnhof, zu wenig und zu teure Parkplätze und vieles mehr. Für nahezu jedes Ärgernis im Alltag außer vielleicht Liebeskummer oder Dauerregen lässt sich mit ausreichend Chuzpe und einer notorischen Streitlust die öffentliche Verwaltung zur Verantwortung ziehen, zumindest versuchsweise, um erstmal sein Mütchen zu kühlen.

\subsection{Kommunalverwaltung auf Aufholjagd}

Kommunalverwaltungen in größeren Städten mit mehreren tausend Beschäftigten lassen sich gut mit komplexeren Konzernen vergleichen. Denn auch in der freien Wirtschaft sind Belegschaften in gewachsenen Strukturen so frei, einen erforderlichen Change-Prozess nicht so ohne weiteres wie das tägliche Mittagsmahl in der Betriebskantine zu verdauen. Personalräte und Gewerkschaften, aber auch mitunter das Führungspersonal tun sich schwer, die Digitalisierung mit ihren Risiken und Nebenwirkungen als Chance zu begreifen, sowohl für die internen Arbeitsprozesse als auch für die Kommunikation nach außen. Dabei lässt sich kommunale Kommunikation mit den digitalen Möglichkeiten effizienter und wirksamer und vor allem offensiver gestalten.

Wie in vielen Kommunen wird auch in der Stadtverwaltung Hannovers schon seit mehr als einem Jahrzehnt von der Digitalisierung gesprochen, ähnlich wie 
in der Bundesregierung. Mit einem Unterschied: Anstelle einer Aufteilung der Kompetenzen auf drei bis fünf Bundesministerien und folglich gegenseitigen Blockaden, erzwingt der kommunale Handlungsdruck durchaus mehr digitalen Fortschritt in den Verwaltungen - wenn auch in kleinen Schritten, so doch beharrlich und allen Bedenkenträgern zum Trotz. So erhielt Hannovers Stadtverwaltung im November 2018 den eGovernment Kommunal Award 2018 der Fachzeitschrift ,eGovernment Computing' für die Entwicklung und Umsetzung digitaler Strategien in der Stadtverwaltung (Landeshauptstadt Hannover 2018a). Die Voraussetzungen dafür schuf der schon erwähnte Stadtdialog „Mein Hannover 2030“. Digitalisierung wurde dort auch in Hannover als ein zentrales Zukunftsthema benannt - und tatsächlich angegangen. Ein Digitalisierungsbeauftragter und ,Digitale Scouts' in den Fachbereichen sorgen für den Know-How-Transfer in die Verwaltung hinein. Ergebnis sind u. a. Digitalisierungsprojekte im Verkehrsmanagement, in der Parkraumsteuerung und im Bürgerservice. Für die Kommunikation nach außen wesentlicher sind jedoch aktuelle Informationskanäle wie die Homepage www.hannover.de, eigene Facebook- und Twitter-Accounts, eine Beschwerde-App oder auch Serviceangebote wie öffentliches WLAN im Rathaus und anderen Dienstgebäuden mit Publikumsverkehr.

Last but not least gehört zu den Rahmenbedingungen kommunaler Kommunikation noch die besondere verfassungsrechtliche Konstellation von Politik und Verwaltung auf der kommunalen Ebene. Sie unterscheidet sich von der Länderund Bundesebene, durchaus mit Auswirkungen auf die Anforderungen der Kommunikation. Vereinfacht ausgedrückt, kommuniziert die Pressestelle einer Stadt sowohl für einen direkt vom Wahlvolk gewählten Bürgermeister respektive Oberbürgermeister, als auch für die gesamte Stadtverwaltung. Die einzelnen Ressortverantwortlichen zum Beispiel für Bauen, Verkehr, Wirtschaft, Umwelt, Finanzen, Bildung, Kultur, Soziales, Sport, Personal etc., die in Hannover Dezernenten oder Stadträte heißen und in der Regel zwei oder drei Fachbereiche führen, sind wiederum von der Ratsmehrheit für acht Jahre gewählt. Das Stadtparlament ist zudem nicht - wie ein Landtag oder der Bundestag - der Legislative zuzuordnen, weil Kommunen anders als Länder oder der Bund keine Gesetze beschließen oder ändern können. Stattdessen sind die gewählten kommunalen Ratsleute staatsrechtlich betrachtet Teil der Exekutive, also der Stadtverwaltung. In der Regel kümmert sich der Rat nicht um die Kommunikation der Stadtverwaltung, auch ist die Kommunikationsabteilung der Verwaltung nicht für die Öffentlichkeitsarbeit der Ratsfraktionen zuständig. Allerdings schaut der Rat bei Personalentscheidungen oder auch Budgetfragen der Kommunikation auf die Finger. 


\section{Chancen nutzen - Kommunale Kommunikation proaktiv und digital}

Die im Zuge der Digitalisierung veränderten Rahmenbedingungen für die kommunale Kommunikation bedeuten zweierlei: Die Machtverschiebung weg vom Informationsanbieter (hier die Kommune) hin zum Nutzer (hier die Bürger) erfordert eine wesentliche aktivere Kommunikation als zu Zeiten analoger Kommunikation. Die klassischen Medien sind bei weitem nicht mehr alleiniger Informationsvermittler. Sie erreichen immer weniger Bürger und müssen zudem ihren Journalismus immer stärker an eigenen Interaktions- und Marketinginteressen ausrichten. Wie behält Kommunikation in den digitalen Erregungswellen den Kopf über Wasser? Ob die Erregung berechtigt oder unberechtigt ist, ob ihr womöglich ,Fake News' zugrunde liegen, interessiert in der Praxis so gut wie gar nicht mehr. Der kommunalen Kommunikation bleibt nichts anderes, als mitzumachen und aktiv die eigenen Kanäle entsprechend offensiv zu nutzen. Broschüren, Faltblätter für die Bürger, Pressemitteilungen und gelegentliche Pressegespräche für geneigte Vertreter der Lokalmedien sind nicht völlig passé, aber längst nur noch ein Teil von kommunaler Kommunikation. Die Existenz ausreichend eigener Online-Kanäle und deren bürgernahe Ausrichtung und kontinuierliche Weiterentwicklung sind heute Mindestvoraussetzungen für eine moderne integrierte kommunale Kommunikation mit den Bürgern.

\subsection{Homepage und Social-Media-Kanäle}

In der Landeshauptstadt Hannover starteten wie in vielen großen deutschen Städten Mitte der 1990er Jahre die Aktivitäten für ein Stadtportal, zunächst privatwirtschaftlich, dann ab 1999 in der Verantwortung der Stadt. Das Portal www. hannover.de bekam bald einen zweiten ,Absender' neben der Stadt: Die 2001 gegründete Region Hannover. Sie ist ein bundesweit einmaliger Kommunalverband mit einer Millionen Menschen, der 21 Städte und Gemeinden einschließlich der Landeshauptstadt umfasst. Während außer Hannover selbst alle anderen kleineren Städte und Gemeinden der Region Wert auf eine eigene Homepage legen, haben sich Landeshauptstadt und Region Hannover unter dem Dach eines Portals versammelt, das zudem die Tochtergesellschaften für Marketing \& Tourismus sowie für Wirtschaftsförderung beheimatet. Folglich ist www.hannover.de ein ebenso starkes wie zugleich überfrachtetes kommunales Portal, obgleich es im Laufe der Jahre mehrfach ,relauncht` wurde. Die Crux: In einem derart umfangreichen 
Portal findet sich alles, nur finden die User oft nicht das, was sie gerade suchen. Und die einzelnen Partner finden sich nicht ausreichend prominent repräsentiert, stoßen sich an vermeintlich klickträchtigen Infos über Bundesliga-Sport und Großkonzerte. Auf der anderen Seite stößt ein Portal, das wie www.hannover.de nur dosiert Werbeplatz anbietet, an finanzielle Grenzen, erst recht angesichts knapper öffentlicher Kassen, auch in wirtschaftlich durchaus gesunden Kommunen.

Ein Internetportal ist aber nur so gut wie seine permanente Erneuerung und deren ausreichende finanzielle Absicherung. Stagnierende und bisweilen sogar rückläufige Nutzerzahlen haben in Hannover die Politik in Stadt und Region 2017 veranlasst, der Geschäftsführung der Hannover.de Internet $\mathrm{GmbH}$ eine Generalinspektion zu finanzieren, unterlegt mit einer Dreiviertelmillion Euro. Vorrangiges Ziel: Mehr Übersichtlichkeit und höhere Auffindbarkeit der Inhalte sowie stärkere Berücksichtigung der ureigenen Inhalte von Stadt und Region. Wer zahlt, bestellt eben auch die Musik, die anschließend gespielt wird.

Das Beispiel Hannover mit einer überfrachteten Homepage zeigt überdies, wie schwer es ist, eine Homepage mit vielen Info-Lieferanten zu verschlanken. Jahrelang wurde in den Städten das eigene Internetportal vom Großteil der Verwaltung als Spielwiese von Computerfreaks angesehen und deren Redaktion nur spärlich mit Inhalten versorgt. Das hat sich im Laufe der Jahre verändert, spätestens mit dem Aufkommen der internetfähigen Smartphones sogar ins Gegenteil verkehrt. Sowohl Anzahl als auch Länge der in der Verwaltung für das Internet erstellten Texte ist angeschwollen. Das Übermaß an Onlinestoff kann kaum kontinuierlich gepflegt werden und ist schnell veraltet. Verlässlichkeit der Information ist jedoch das A und O: Die Öffnungszeiten eines Bürgeramtes müssen stimmen. Jede außerplanmäßige Schließung muss auf der entsprechenden Seite hinterlegt sein, inklusive einer Notfall-Hotline für eilige Angelegenheiten. Je höher die Aktualität ist, desto wichtiger sind die schnellen Social-Media-Kanäle. Die eintägige SchlieBung einer Behörde, die Änderung der Öffnungszeit einer Stadtteilbibliothek oder anderweitige Service-Informationen müssen also nicht nur auf der Homepage platziert werden. Die steigende Zahl der Fans und Follower von Facebook und Twitter können zudem unmittelbar angesteuert werden, während Informationen auf der Homepage von den Adressaten erst aktiv gesucht werden müssen.

So betreibt die Landeshauptstadt Hannover eigene Accounts - in diesem Fall unabhängig von der Region Hannover - bei Facebook seit Juni 2013 und bei Twitter seit Juli 2014. Dies geschieht im Übrigen mit Duldung durch den behördlichen Datenschutzbeauftragten, was keine Selbstverständlichkeit ist. Facebook und Twitter halten sich bekanntermaßen nicht an deutsche Datenschutzgesetze. Der tolerierte Kompromiss besagt, dass ein Nutzer von anderen Online-Veröffentlichungen wie etwa auf der Homepage www.hannover.de nie automatisch auf die 
Social-Media-Portale wechseln kann. Aufgrund der Datenschutz-Problematik geraten Behörden bei Social-Media-Kanälen strukturell auch deshalb schnell ins Hintertreffen, weil das Freischalten einer neuen Plattform in der Regel einen verwaltungsinternen Abstimmungsprozess voraussetzt, der durchaus seinen Sinn hat, mit dem Digitalisierungstempo aber selten Schritt halten kann. Mit 21.000 Facebook-Fans und 14.500 Twitter-Follower (Ende 2018) bleibt Hannovers Stadtverwaltung in den Sozialen Medien noch deutlich hinter den Lokalzeitungen HAZ und der kleineren Neue Presse zurück.

Gleichwohl ist insbesondere der Twitter-Kanal von strategisch hoher Relevanz. Mittels Twitter als News-Kanal verfügen Kommunalverwaltungen erstmals über ein Instrument, Bürger und Medien zeitgleich und in Echtzeit zu informieren. Die Durchschlagskraft von Twitter ist vor allem in Großlagen evident: Bombenräumungen, Großbrände, Anschläge und andere Katastrophen- oder Großlagen, wo Kommunikation schnell in den Krisenmodus schalten muss. Hier ist Twitter der Kanal, der einer Kommunalverwaltung die Informationshoheit von Beginn an sichern oder in Krisensituationen zumindest schnell wiedererlangen hilft. Drei Beispiele aus Hannover, Frankfurt und München belegen dies eindrucksvoll.

\subsection{Beispiel Hannover - Bombenräumung im Realtime- Newsfeed}

Ein Jahr nach dem Start bei Twitter entschied sich die Kommunikation in Hannover zusammen mit der städtischen Feuerwehr erstmals zu einer Live-Information über diesen Kanal. Der Anlass: Bei Bauarbeiten mitten in einem Wohnviertel nahe der City war eine große Fliegerbombe aus dem Zweiten Weltkrieg freigelegt worden, die noch am selben Tag entschärft werden sollte. Dies bedeutete die größte Bombenräumung in der Geschichte Hannovers nach dem Zweiten Weltkrieg: 31.000 Menschen mussten ihre Wohnungen binnen weniger Stunden verlassen. Der erste Tweet der Stadt dazu am 19. Mai 2015 lautete:

„\#Eilmeldung: Fliegerbombe in der \#Südstadt freigelegt, Entschärfung des Blindgängers noch heute. Mehr in Kürze! \#hannbombe\#hannover ${ }^{\wedge} \mathrm{MH}{ }^{\wedge}{ }^{\wedge} W^{“}$ (Stadt Hannover 2015)

Um alle Infos zur Bombenräumung von Stadt, Medien und Privatleuten zu kanalisieren und für alle gleichermaßen leicht aufrufbar zu machen, schlug die Redaktion der Stadtverwaltung den gemeinsamen Hashtag \#hannbombe vor. Die 
Steuerungsfunktion dieses Hashtags wurde von allen akzeptiert - und ist seither bei jeder Bombenräumung in Hannover Standard. Per Twitter lassen sich alle wichtigen Etappen zur Bombenentschärfung aktuell und präzise veröffentlichen und über die Vervielfältigung durch Medien und private User in Windeseile breit streuen. Herkömmliche Kanäle über Rundfunk und Lautsprecherdurchsagen in den Wohnvierteln werden unverändert genutzt.

Während der ersten Twitter-begleiteten Bombenräumung waren Reporter der Medien zunächst irritiert. Nach Twitter-News der Stadt fragten sie noch einmal per Telefon beim Sprecher der städtischen Feuerwehr nach, ob dies auch so richtig sei. Da die Kurznachricht per Twitter eine offizielle Mitteilung der Stadt war, bedurfte es eigentlich keines solchen weiteren Anrufs. Inzwischen ist Twitter als der maßgebliche Erstinfokanal bei besonderen Großereignissen gelernt und akzeptiert. Da direkt aus der Leitstelle der Feuerwehr heraus im Vier-Augen-Prinzip getwittert wird, kommen die Infos im ,Realtime"-Modus, wie dies lange Zeit nur bei Börsenkurs-Informationen praktiziert wurde. Schneller, verlässlicher und damit auch glaubwürdiger lässt sich behördliche Kommunikation auch auf kommunaler Ebene nicht gewährleisten.

\subsection{Beispiel Frankfurt - Fataler Fehlalarm durch fehlende Information}

In Frankfurt führte vor wenigen Jahren eine via Facebook verbreitete Fehlinterpretation eines Feuerwehreinsatzes durch einen Augenzeugen die Notwendigkeit einer sehr aktiven und frühen öffentlichen Gegensteuerung eindringlich vor Augen. Aus dem 20. Stock eines Hochhauses wurde per Notruf ein normaler Wohnungsbrand gemeldet. Wegen der Höhe rückte die Feuerwehr mit größerer Ausrüstung an. Eine Privatperson machte bei Einsatzbeginn sofort ein Foto und postete dies auf Facebook. Dieses Bild - augenscheinlich ein Großeinsatz an einem Hochhaus, aus dem es hoch oben qualmt - verselbstständigte sich im Netz zu der Nachricht, dass es in Frankfurt wohl einen Anschlag nach dem Muster des 11. Septembers 2001 in New York gegeben habe. Diese Spekulation wurde anschließend noch genährt, weil die Pressestelle der Feuerwehr auf erste Medienanfragen zu dem ,Anschlag ' nichts klarstellen konnte. Sie kannte den harmlosen Einsatz noch nicht. Von einem solchen Wohnungsbrand wäre die Pressestelle wie üblich erst nach dem Löschen informiert worden.

Die Konsequenz daraus: Social-Media-Kanäle werden seither permanent im Auge behalten, nicht nur bei eigenen Einsätzen. Nur so können Feuerwehr oder Polizei sofort reagieren und gefährliche Spekulationen richtigstellen, ihre 
Wirkung eindämmen und die Informationshoheit zurückholen. Denn jeder größere Einsatz wird heute in Echtzeit live im Netz begleitet - durch die User. Das beginnt mit Live-Fotos von Großbränden, Anschlägen oder Unfällen, die schon entstehen, bevor überhaupt Einsatzkräfte vor Ort sind. Ein erfahrener Einsatzleiter schildert sehr eindringlich die Folgen für die Kommunikation:

\begin{abstract}
„Die Erstbilder sind fatal. Meist sind noch keine Rettungskräfte zu sehen, weil diese erst nach wenigen Minuten eintreffen. Sobald solche Bilder im Netz kursieren, lösen sie kritische Reaktionen bis zu Panik aus. Der digitale Bürger reagiert sofort, fragt bei Freunden nach, dann bei Polizei und Feuerwehr - und blockiert die Notrufnummern 110 und 112 genau in der Frühphase eines Großereignis, wo Rettungsleitstellen sich erst eilig darauf einstellen müssen."
\end{abstract}

Abhilfe schafft nur eine mindestens ebenso aktive Social-Media-Kommunikation, die von Beginn an bei jedem Großereignis läuft, die unmittelbar mitgedacht werden kann, weil die Ressourcen bereitstehen und die Abläufe geübt sind.

\title{
3.4 Beispiel München - Per Twitter Panik eingedämmt
}

Wie sich selbst in einer extremen Gefahrensituation in einer Großstadt mit völlig ungewissem Ausgang die Bevölkerung etwas beruhigen und steuern lässt, zeigt die Kommunikation der Münchner Polizei anlässlich des Amoklaufes am 22. Juli 2016. Ein 18-Jähriger tötete am und im Olympia-Einkaufszentrum (OEZ) im Stadtteil Moosach neun Menschen. Fünf weitere verletzte er durch Schüsse. Die Polizei war mit einem Großaufgebot im Einsatz. Um 17.52 Uhr ging der erste Notruf bei der Münchner Polizei ein: „Schüsse im OEZ“. Kurz darauf gab es schon die ersten privaten Tweets auf Twitter. Rund 200.000 Nachrichten zu diesem Amoklauf folgten in dieser Nacht allein auf Twitter. Die Polizei München schaffte es mit ihren Twitter-Infos sehr schnell, an die Spitze dieser gigantischen Informationswelle zu gelangen.

Mit mittlerweile nach eigenen Angaben mehr als zwölf Millionen Usern monatlich in Deutschland ist Twitter ein sehr ernstzunehmender Nachrichtenkanal. Anders als bei Facebook werden Nachrichten nicht nach undurchschaubaren Algorithmen gefiltert und nur einem Teil der Nutzer gezeigt. Während des Amoklaufs in München gab es teils mehr als 1000 Tweets in der Minute. Die Münchner Polizei mit ihrem Social-Media-Team kommunizierte zunächst fast nur noch über Twitter und dominierte mit 113 Tweets in Deutsch, Englisch und Französisch die Informationslage in dieser Nacht. Sie informierte, warnte, stellte richtig, 
beruhigte und ermahnte gelegentlich, dass manche Bilder und Videos eher dem Täter helfen könnten. Die Polizei-Tweets wurden am häufigsten weiterverbreitet und rund 100 Mio. Mal gesehen.

Verunsicherung und Panik-Reaktionen sind die Folge, wenn Polizei und Feuerwehr sich nicht schnell über dieselben Kanäle Gehör verschaffen. Das erste private Live-Video eines Amateurs in München ohne jede offizielle Bestätigung erreichte 125.000 Menschen. Es gibt in solchen Situationen schnell gefälschte Informationen. So kursierte während des Amoklaufes im Netz plötzlich ein Foto, das angeblich Verletzte an einer Rolltreppe in dem betroffenen Münchner Kaufhaus zeigte. Das Foto war in Wahrheit ein Jahr zuvor in Südafrika aufgenommen worden. Ein TV-Sender verbreitete das Fake-Foto bereits, ehe es offiziell entlarvt wurde. Das Fazit aus Sicht der Kommunikation nach dem Münchner Amoklauf: Behörden müssen in solchen Situationen präsent sein, sie können und müssen glaubhaft informieren und somit auch eine potenzielle Paniksituation wieder in den Griff bekommen.

\subsection{Deeskalierende Kommunikation und Beschwerdemanagement}

Die schnellen sozialen Netze stärken die Medienmacht jedes Bürgers, der digital kommuniziert. Abseits von Großlagen zeigt dies die schon skizzierte Zunahme von Beschwerden und Hassmails. Eine aktive Kommunikationsstrategie gelingt hier dennoch wesentlich schwieriger als in extremen Lagen wie Bombenräumungen oder Amokläufen. Dieser Umstand mag auf den ersten Blick erstaunlich sein, erklärt sich jedoch bei näherem Hinsehen, insbesondere als ,Haltungsproblem‘. Denn diese Beschwerden und Drohungen werden vor allem als ungerecht und lästig empfunden, was sie zweifellos in der Regel zu einem großen Anteil auch sind. Doch selbst die in pauschalem Absolut vorgetragenen Beschwerden der Güte ,Flüchtlinge bekommen alles. Wir bekommen nichts!" oder ,Die Verwaltung verspricht viel. Aber sie kümmert sich nicht!' haben eine Appell-Funktion. Sie weisen bei allem Gedröhne und Gewüte darauf hin, dass jemand etwas gänzlich falsch verstanden hat oder falsch verstehen möchte und laden letztlich doch zur Kommunikation ein, zumindest wenn der Absender sich namentlich outet. Nur wer seine Identität nicht preisgibt und anonym die Kommunalverwaltung angeht, will meist nicht mehr kommunizieren.

Alle anderen aber sollten nicht von vornherein aufgegeben werden. Denn die als lästig empfundene Anfrage kann bei schlechter Reaktion zu einem öffentlichen kommunikativen Problem werden. Doch wie lässt sich auf derlei Anwürfe 
reagieren? Zunächst einmal heißt es, die Beschwerden und Vorwürfe im Kern ernst nehmen, weniger die Gefühle spielen lassen, mehr den Verstand und Haltung zeigen. Eine souveräne und sachliche Herangehensweise lässt leichter Mängel oder Probleme eingestehen, erzeugt eine eher wertfreie, erklärende Sprache und vermittelt glaubhaft Empathie und Offenheit und Interesse an Abhilfe. Wenn eine Beschwerde haltlos ist, hat erst recht nur eine souveräne sachliche Reaktion die Aussicht auf Verständnis. Im Kern geht es also um die Haltung in Sachen Konfliktfestigkeit und Konfliktfähigkeit. Diese sind Voraussetzung für eine offene zunächst interne Kommunikation über den Problemfall, die anschließend in eine souveräne Kommunikation nach außen an die Beschwerdeführer und bei Bedarf auch an die Medien mündet.

Eine solche Haltung macht den Blick frei für richtige Reaktionen: Beschwerden ernst nehmen, schnelle erste Rückmeldung, dass die Angelegenheit umgehend bearbeitet wird, Wiedervorlage für das interne Nachfassen, finale Rückmeldung. Dies kann auch mal in eine kluge Umarmungsstrategie münden, wie das Beispiel einer ostdeutschen Großstadt zeigt, wo der Oberbürgermeister notorische Nörgler gern zum Gespräch bei Kaffee und Kuchen einlädt. Wer kommt, ist schon von dem Gesprächsangebot beeindruckt und neigt zu deutlich sachlicherem Ton. Wer nicht kommt, ist mutmaßlich noch mehr beeindruckt und meldet sich vorerst nicht mehr.

Auch bei denjenigen, die vornehmlich anonym pöbeln, drohen und nicht mehr kommunizieren wollen, hilft eine souveräne Haltung, um dann je nach Medium entsprechend $\mathrm{zu}$ agieren: sei es freundlich, aber bestimmt um einen anderen Ton zu bitten, öffentliche Pöbeleien auf eigenen Kanälen zu löschen oder sachlich richtig zu stellen, oder sei es im Zweifel im Extremfall auch nicht vor Anzeigen zurückzuschrecken.

Gerade die sozialen Netze sind dafür geeignet, regelrechte Kampagnen gegen eine Kommunalverwaltung zu fahren. In einer Stadt wie Hannover, deren Oberbürgermeister seit dem Zweiten Weltkrieg ausnahmslos Sozialdemokraten sind, weil dies vom Wahlvolk mehrheitlich so gewollt ist, greifen Kritiker, sobald etwas schiefläuft, gern zum Filz-Vorwurf. Für die Kommunikation einer solcherart abgestempelten Kommunalverwaltung bedeutet dies in Zeiten schneller digitaler Kanäle, dass diese tatsächlich permanent im Auge behalten werden müssen. Ein solches Monitoring nicht nur der eigenen Kanäle ermöglicht potenziell brisante Themen frühzeitig zu identifizieren und auf dieser Basis eine ,Gegen'-Kommunikation zu entwickeln. Die Stadt Heidenheim in Baden-Württemberg hat zum Beispiel hierfür eine intern für alle einsehbare ,Social Wall` eingerichtet, auf der alle im Netz kursierenden relevanten Themen präsent sind. In dem Maße, wie 
eine Verwaltung über die relevanten kommunalen Diskussionen im Netz informiert ist, ist sie auch in der Lage, souverän und schnell zu reagieren.

Dies setzt allerdings voraus, dass alle Bereiche, die nach außen kommunizieren, voneinander wissen. Beschwerden von Bürgern landen in der Regel jedoch nicht bei der Abteilung für Presse- und Öffentlichkeitsarbeit, sondern im Bürgerbüro, direkt beim Oberbürgermeister als Verwaltungschef oder auch direkt bei den zuständigen Fachbereichen. Allein diese unterschiedlichen Adressaten wissen in der Regel nicht automatisch etwas von ähnlichen Beschwerden bei anderen. Erst recht ist die Kommunikationsabteilung im Normalfall nicht eingeschaltet, solange Medien von der Angelegenheit keinen Wind bekommen. Werden sie von Beschwerdeführern jedoch eingeschaltet, kommen prompt die Anfragen. Wenn die Pressestelle noch keinen Schimmer von dem Fall hat, läuft sie schnell in die Kommunikationsfalle. Viele Kommunen haben im Laufe der Jahre ein Beschwerdemanagement aufgebaut. Die Vernetzung mit dem Kommunikationsbereich allerdings zählt dabei noch zu den Stiefkindern. Das Zusammenwachsen von Medienkommunikation, behördlicher und privater Kommunikation in den digitalen Medien macht es jedoch notwendig, dass Beschwerden mit öffentlichkeitswirksamem Potenzial in einer Kommunalverwaltung alle potenziellen Kommunikatoren frühzeitig erreichen, damit die Reaktion nach innen und nach außen abgestimmt und transparent in alle Kanäle geht.

\section{$4 \quad$ Keine kommunale Kommunikation ohne Beteiligung}

Die beste Kommunikationsstrategie der Presseabteilung hilft wenig, wenn die politisch-administrativen Grundlagen schlecht sind, wenn Vorhaben der Kommunalverwaltung der Bevölkerung auf allen Kanälen via Medien, Homepage, Facebook, Twitter und in öffentlichen Versammlungen nach Beschluss lediglich verkündet werden. Im nicht mehr so ganz jungen 21. Jahrhundert bleibt der erhoffte Applaus dann oftmals aus. Die Wahl für fünf Jahre sichert der regierenden Mehrheit besonders auf der kommunalen Ebene mitnichten mehr fünf Jahre kommentar- und kritiklose Unterstützung. Jedes komplexere Vorhaben bedarf heute einer Legitimation mittels einer Bürgerbeteiligung. Fehlt es daran, müssen zuvorderst die Kommunikationsabteilungen der Kommunen diese Defizite ausbaden. Bürgerprotest liefert Lokalmedien begehrten Konfliktstoff. Spätestens seit den Auseinandersetzungen um den Bahnhofsneubau Stuttgart 21 hat sich diese Erkenntnis durchgesetzt: Ohne eine gute Beteiligung gibt es keine gute Kommunikation und keine erfolgreiche Politik. 


\subsection{Glanz und Elend: Beteiligen, aber wie?}

Auch weniger spektakuläre Beispiele als das Stuttgarter Milliardenprojekt verdeutlichen, wie leicht eine Kommunalverwaltung trotz bester Absichten massiv in die Kritik gerät, weil Bürger sich übergangen fühlen. So löste in einer Kleinstadt in Rheinland-Pfalz die Information der Pressestelle über die Fertigstellung eines geringfügig verlegten Radweges völlig überraschend einen Shitstorm im Netz aus. Plötzlich wurde Unverständnis für die Verlegung laut; das eigentlich als sinnvoll erachtete Projekt wurde als „Fehlplanung“ und „Verschwendung von Steuergeldern“ gegeißelt, alternative Vorschläge machten die Runde. Möglicherweise hätte sich bei einer frühzeitigen Einbeziehung betroffener und/oder interessierter Bürger am Ende auch die umgesetzte Variante als die letztlich beste herausgeschält. Allerdings wäre sie dann positiv aufgenommen und nicht in Bausch und Bogen verrissen worden.

Beteiligungsprozesse haben zudem eine gewisse Halbwertzeit. Was einmal Konsens oder mehrheitsfähig zu sein schien, muss es nach einigen Jahren nicht mehr sein. Diese Erfahrung musste das Baudezernat der Landeshauptstadt Hannover bei ihren Plänen zur überfälligen Umgestaltung des Steintorplatzes machen. Das Vorhaben war in einem intensiven Beteiligungsprozess zur Innenstadtentwicklung City 2020 in den Jahren 2008/2009 schon einmal angesprochen worden, gehörte aber abschließend nicht zu den als vorrangig identifizierten Orten. Nach und nach ging das Baudezernat die Umgestaltungen mehrerer anderer Plätze an, zum Teil mit zusätzlichen Bauten für Büros und Wohnraum, alles insgesamt mit positiver Kommunikation. Schließlich geriet dann bei den Planern der Behörde auch der Steintorplatz wieder in den Blick. Doch nun wurden Stimmen laut, die vor einer weiteren intensiven Bebauung von Innenstadtplätzen warnten. Ein erneutes Beteiligungsthema wurde der Steintorplatz jedoch nicht, da aus Sicht des Dezernates alles bereits diskutiert worden war - dies lag allerdings fünf Jahre zurück.

Im Vorfeld der Kommunalwahlen im Herbst 2016 machten dann Anwohner des Steintorplatzes im Verein mit Opposition und notorischen Kritikern der Stadtpolitik die mittlerweile weitgediehenen Steintorpläne des Baudezernats zum dominierenden Thema des Wahlkampfes. Es drängte auch den Zustrom von weit mehr als 10.000 Flüchtlingen binnen weniger Monate, den die Stadtverwaltung im Verein mit ehrenamtlichen Helfern - wie in vielen Kommunen - bravourös bewältigte, in den Hintergrund. Spät organisierte die Bauverwaltung auf Anraten der Kommunikation noch eine Informationsveranstaltung, der aber bereits andere Veranstaltungen u. a. auch seitens der führenden Lokalzeitung HAZ vorangegangen waren. 
Bei den Kommunalwahlen verloren dann SPD und Bündnis 90/Die Grünen ihre fast drei Jahrzehnte bestehende Mehrheit im Stadtrat. Seither sitzt die FDP als dritter Bündnispartner mit am Tisch. Die Wahlanalysen verwiesen maßgeblich auf das Problemthema Steintorplatz. Hannovers Oberbürgermeister stoppte bereits am Tag nach der Wahl die aktuellen Planungen und kündigte eine neuerliche Beratung in der Politik und zusammen mit den Bürgern zur Zukunft des Steintorplatzes an. Zwei Jahre später startete dann eine über mehrere Monate angelegte umfangreiche Bürgerbeteiligung (Landeshauptstadt Hannover 2018b).

Dieses Beispiel zeigt, dass auch eine Stadt, die wie Hannover seit den 1970er Jahren vielfältige Beteiligungsformate entwickelt hat, dennoch nicht gegen fatale Fehleinschätzungen gefeit ist. So gelang beispielsweise 1992 mit einem stadtweiten Bürgerentscheid mit knappem Ausgang die Befriedung der Stadt zur höchst umstrittenen Ausrichtung der ersten Weltausstellung in Deutschland, der Expo 2000. Im Jahr 2010 baute Hannover als erste deutsche Großstadt für schnelle repräsentative Online-Befragungen zu wichtigen Stadtthemen ein sogenanntes Bürgerpanel mit mehr als 2500 Bürgern auf - nach Alter und Geschlecht repräsentativ zusammengesetzt (Masser und Möser 2014). Mehr als drei Viertel der Befragten machen online mit, ein knappes Viertel bevorzugt den Postweg, mit abnehmender Tendenz. Seither gab es ein halbes Dutzend Befragungen zu Mobilität, außerschulischen Bildungseinrichtungen, Sportförderung, zum Stadtdialog 2030, zum ehrenamtlichen Engagement und zuletzt zur Sicherheit und Ordnung in der Stadt - stets mit einer Beteiligung von mindestens zwei Dritteln des Panels und ausführlicher Berichterstattung der Ergebnisse in den Lokalmedien (Hogan et al. 2018).

Wir können festhalten: Beteiligung sichert politische Entscheidungsprozesse $\mathrm{ab}$ und erleichtert Kommunikation, weil sie Blockaden und Vorbehalte abbaut und Entscheidungen transparenter macht. Dass Bürgerbeteiligung auch in digitalen Zeiten nicht vorrangig online funktioniert, sondern das klassische Gespräch offline und real unverändert kommunikativ höchst wertvoll ist, zeigt abschließend das Beispiel Heidelberg.

\subsection{Beispiel Heidelberg - Vorreiter für mitgestaltende Bürgerbeteiligung}

Heute gibt es in Städten und Gemeinden so gut wie keine öffentliche Veranstaltung, in der nicht „mehr Beteiligung“ oder „mehr Partizipation“ gefordert wird (Leggewie und Nanz 2016, S. 27 f.). Dies bleibt zwar vielfach noch ein Lippenbekenntnis, dennoch haben sich mittlerweile mehr als 70 Kommunen Leitlinien für Bürgerbeteiligung 
verordnet, die mehr oder minder gut gelebt werden (Netzwerk Bürgerbeteiligung 2018). Eine Vorreiterrolle spielt dabei Heidelberg.

Die traditionsreiche Universitätsstadt im Südwesten mit rund 160.000 Einwohnern beschloss 2012 als eine der ersten deutschen Großstädte nicht nur sehr konkrete und verbindliche „Leitlinien für die mitgestaltende Bürgerbeteiligung“ (Stadt Heidelberg 2015). Sie koppelte dies mit einer fortan laufenden Veröffentlichung aller wesentlichen Vorhaben der Stadtverwaltung. Bereits 2013 waren gut die Hälfte der Bürger zufrieden mit den angebotenen Möglichkeiten für Beteiligung. Mittlerweile ist die Zufriedenheit auf $60 \%$ gestiegen, nur rund $20 \%$ halten das Angebot für nicht ausreichend, wie der jüngste Evaluationsbericht ausweist (Stadt Heidelberg 2018).

Ende 2018 waren 119 laufende Vorhaben auf der Heidelberger Vorhabenliste erfasst, das älteste noch nicht abgeschlossene stammte aus dem Dezember 2015. Davon ist bei mehr als zwei Dritteln (68\%) angegeben, dass sie mit Bürgerbeteiligung stattfinden, das heißt, Bürger werden einmal oder wiederholt in verschiedenen Phasen des Projektes beteiligt. Die Vorhabenliste steht digital zur Verfügung; wer will, kann sich jede Aktualisierung per E-Mail von der eigens geschaffenen Koordinierungsstelle Bürgerbeteiligung anzeigen lassen. Ihr Leiter Frank Zimmermann sieht als wesentliche Voraussetzung für das Gelingen kommunaler Beteiligung verbindliche Regeln: „Die Spielregeln sind in Heidelberg völlig klar und akzeptiert. Sie müssen transparent und stimmig sein und vom Gemeinderat beschlossen werden. Ohne Prozesslegitimation gibt es auch keine Ergebnislegitimation." So wie die Themen in jeder Stadt spezifisch sind, so wenig reicht es, Leitlinien anderer Städte einfach zu übernehmen. „Jede Kommune muss sich ihre Spielregeln selbst erarbeiten. Nur so schafft sie eine hohe Verbindlichkeit und Klarheit", empfiehlt Zimmermann. Erfolg hat zudem nur, wer früh beteiligt. „Und Beteiligung muss ernst gemeint sein, ein echter Dialog“, rät Zimmermann, der sich als Koordinator der Interessen von Politik, Bürgern und Verwaltung naturgemäß meist „Zwischen allen Stühlen“ sitzen sieht. Spätestens nach Abschluss eines Beteiligungsprozesses muss dokumentiert und auch für Nicht-Beteiligte nachvollziehbar kommuniziert werden, wie es zu den Ergebnissen kam und welches die zentralen Argumente im Beteiligungsprozess waren. Nur so wird deutlich, warum nicht alle Konflikte ausgeräumt werden können und wie Verwaltung und Politik nach Abwägen aller Argumente ihre Entscheidungen getroffen haben. Völlig konfliktfreie und einvernehmliche Konsenslösungen sind in der Regel kaum möglich. Doch Frank Zimmermann beschreibt sehr anschaulich den demokratiestiftenden Zweck von Beteiligung: 
„Als sogenannter Wutbürger bin ich ein Bürger, der nicht gehört wurde, und deshalb wütend werde, weil ich annehme, es wäre anders geworden, wenn meine Gedanken gehört worden wären. Wenn ich aber gehört werde und gleichzeitig selber höre, dass andere vielleicht etwas ganz anderes wollen, und wenn dann am Ende etwas herauskommt, das mir nicht so gefällt, kann ich es vielleicht nur schwer akzeptieren, werde aber nicht mehr wütend.“

Das Heidelberger Modell schafft dem Evaluationsbericht von 2018 zufolge bei allen mehr Zufriedenheit, mehr Verständnis und mehr Zustimmung für Entscheidungen, selbst wenn nicht alle Interessen befriedet werden können. Der Bericht stellt allerdings auch fest, wo es noch hakt: Zum Beispiel bei Einbeziehung von Gruppen, die sich gemeinhin weniger um Stadtpolitik kümmern, wie so genannte bildungsferne Menschen oder Zugewanderte.

Aus einer aktiven Beteiligungsstrategie ergibt sich folgerichtig auch eine aktive Kommunikationsstrategie. „Man muss alle Kanäle bespielen“, sagt Koordinator Zimmermann. Das sind vor allem die eigenen Kanäle vom traditionellen Heidelberger Stadtblatt an alle Haushalte bis zu den sozialen Medien. Multiplikatoren sind vor allem auch die von Projekten betroffenen Gruppen. Zu klären ist daher rechtzeitig, wer bei einem neuen Beteiligungsprojekt eigentlich alles die Akteure sind und wie diese ihre Kanäle gut nutzen können. Die Lokalmedien selbst sind bei der Kommunikation nur ein Adressat unter vielen. Sie berichten zwar gern über Kritik an zu wenig Beteiligung, über die dann tatsächlich stattfindende Bürgerbeteiligung jedoch eher weniger. Für Medien sind gemeinhin Konflikte der spannendere Stoff. Beteiligung jedoch hat das Ziel, Konsens und Verständnis zu stiften.

\section{$5 \quad$ Conclusio}

Die Praxis kommunaler Kommunikation in Zeiten der Digitalisierung ist gezeichnet von rapiden Veränderungen der Rahmenbedingungen gleichermaßen für Politik und Verwaltung, die klassischen Lokalmedien, Bürger und die zwischen allen agierenden Kommunikationsteams. Internet und die sozialen Netze haben die Kommunikationslage grundlegend verändert.

Die bisherigen Adressaten der Kommunikation durch Medien oder Verwaltung sind zunehmend selbst aktiv. Die genannten Beispiele aus Hannover und anderen Städten zeigen, dass Behörden nur dann erfolgreich kommunizieren, wenn sie diese Realität annehmen und aktiv eingreifen. Anderenfalls werden sie nicht nur digital, sondern auch real überrollt, wie das Scheitern einer mal gewollten und an sich sinnvollen Umgestaltung des Steintorplatzes in Hannover zeigt. Kommunale 
Kommunikation muss alle Kanäle bespielen, vor allem bespielen wollen. Wer Angst vor der Auseinandersetzung in den öffentlichen sozialen Kanälen oder mit noch so wüster Kritik hat, steht automatisch mit dem Rücken an der Wand. Kommunale Kommunikation braucht aber Bewegungsfreiheit, um in jeder Lage vorne sein zu können. Die Instrumente dafür liegen mit der Digitalisierung bereit. Es gilt sie aktiv und strategisch zu nutzen.

Beispiele der Kommunikation in Großlagen mehrerer Großstädte machen deutlich, wie es auf Twitter als derzeit schnellstem Infokanal gelingt, sich an die Spitze der aktuellen Kommunikation zu schieben, wenn nicht sogar von vornherein vorne $\mathrm{zu}$ sein. Eine aktive Kommunikationsstrategie schafft am ehesten Transparenz und Offenheit und damit Vertrauen. Wer sich einmauert und in Abwehrhaltung geht, nährt Misstrauen.

Eine aktive Kommunikation deeskaliert und geht auf Kritik ein, solange Kritik nicht anonym bleibt oder in Hass-Rhetorik ausartet. Wenn nötig, ist der Staatsanwalt gefragt. Auch das gehört in letzter Konsequenz zu einer selbstbewussten Haltung. Kommunale Kommunikation bleibt also dann handlungsfähig, wenn eine Verwaltung die Erregungspotenziale kommunalpolitischer Themen von Beginn an mitdenkt. Sie braucht ausreichend Empathie und Mut und insofern auch politische Rückendeckung, um sich der Ungewissheit digitaler Kommunikationsplattformen und ihrer schwer kalkulierbaren Dynamik zu stellen. Sie muss gerade deshalb eine frühzeitige Bürgerbeteiligung als notwendigen Teil der Öffentlichkeitsarbeit beinhalten. Denn es gilt: Ohne Beteiligung keine gute Kommunikation und ohne Kommunikation keine gute Beteiligung. Eine aktive Kommunikation geschieht auf allen Kanälen, sowohl digital als auch weiterhin offline. Sie gelingt nur, wenn auf allen Kanälen sprichwörtlich ,mit einer Stimme' gesprochen wird. Und dennoch kann sie nicht jede notorische Nörglerin und nicht jeden Querulanten erreichen. Dann gilt es, Gelassenheit walten zu lassen getreu dem Leitsatz der langjährigen Pressechefin einer NRW-Kommune: „Die Taube wird auch weiterhin aufs Spielfeld scheißen.“

\section{Literatur}

Hogan, K., Masser, K., \& Möser, A. (2018). Praxisbeispiel Hannover: Online-gestütztes Bürgerpanel. In Stiftung Mitarbeit \& ÖGUT (Hrsg.), Bürgerbeteiligung in der Praxis. Ein Methodenhandbuch (S. 82-83). Bonn: Stiftung Mitarbeit.

Kaube, J. (2018). Wenn man über alles schreibt, wie bleibt man dann interessant. In P. Felixberger \& A. Nassehi (Hrsg.), Kursbuch 195 (S. 78-97). Hamburg: Kursbuch Kulturstiftung. 
Kruse, J. (2010). Kurzprotokoll Enquete-Kommission Internet und digitale Gesellschaft. 4. Sitzung. Berlin: Deutscher Bundestag.

Landeshauptstadt Hannover. (2018a). Digitalisierungsstrategie der Verwaltung ausgezeichnet. Presseinformation. https://www.hannover.de/Service/Presse-Medien/Landeshauptstadt-Hannover/Meldungsarchiv-f\%C3\% BCr-das-Jahr-2018/ Digitalisierungsstrategie-der-Verwaltung-ausgezeichnet. Zugegriffen: 15. Jan. 2019.

Landeshauptstadt Hannover. (2018b). Der Steintor-Dialog. https://www.steintor-dialog.de/. Zugegriffen: 13. Jan. 2019.

Leggewie, C., \& Nanz, P. (2016). Die Konsultative. Mehr Demokratie durch Bürgerbeteiligung. Berlin: Klaus Wagenbach.

Masser, K., \& Möser, A. (2014). Bürger-Panel Hannover. In H. Hill, M. Martini, \& E. Wagner (Hrsg.), Transparenz, Partizipation, Kollaboration. Die digitale Verwaltung neu denken (S. 181-202). Baden-Baden: Nomos.

Netzwerk Bürgerbeteiligung. (2018). Qualitätskriterien Bürgerbeteiligung. https://www. netzwerk-buergerbeteiligung.de/kommunale-beteiligungspolitik-gestalten/qualitaetskriterien-buergerbeteiligung/. Zugegriffen: 15. Jan. 2019.

Neuberger, C. (2018). Entfesselte Kontexte. In P. Felixberger \& A. Nassehi (Hrsg.), Kursbuch 195 (S. 31-52). Hamburg: Kursbuch Kulturstiftung.

Scheller, J., \& Ullrich, W. (25. Mai 2018). Der Geist zwitschert, wo er will. ZEIT.

Stadt Hannover. (2015). Tweet vom 19. Mai 2019. https://twitter.com/hannover/status/600608225688481792. Zugegriffen: 13. Jan. 2019.

Stadt Heidelberg. (2015). Leitlinien für mitgestaltende Bürgerbeteiligung in Heidelberg. https://www.heidelberg.de/hd,Lde/34712.html. Zugegriffen: 18. Jan. 2019.

Stadt Heidelberg. (2018). Gesamtbericht Evaluation Bürgerbeteiligung 2018. https://www. heidelberg.de/hd,Lde/HD/Rathaus/evaluation+2018.html. Zugegriffen: 18. Jan. 2019.

Open Access Dieses Kapitel wird unter der Creative Commons Namensnennung 4.0 International Lizenz (http://creativecommons.org/licenses/by/4.0/deed.de) veröffentlicht, welche die Nutzung, Vervielfältigung, Bearbeitung, Verbreitung und Wiedergabe in jeglichem Medium und Format erlaubt, sofern Sie den/die ursprünglichen Autor(en) und die Quelle ordnungsgemäß nennen, einen Link zur Creative Commons Lizenz beifügen und angeben, ob Änderungen vorgenommen wurden.

Die in diesem Kapitel enthaltenen Bilder und sonstiges Drittmaterial unterliegen ebenfalls der genannten Creative Commons Lizenz, sofern sich aus der Abbildungslegende nichts anderes ergibt. Sofern das betreffende Material nicht unter der genannten Creative Commons Lizenz steht und die betreffende Handlung nicht nach gesetzlichen Vorschriften erlaubt ist, ist für die oben aufgeführten Weiterverwendungen des Materials die Einwilligung des jeweiligen Rechteinhabers einzuholen.

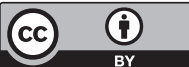

